\title{
SECOND-ORDER LINEAR SYSTEMS WITH SUMMABLE COEFFICIENTS*
}

\author{
BY

\section{J. H. STURDIVANT}

Sturm and his successors $\dagger$ have examined in detail properties of the zeros of solutions of linear differential systems similar to (1), (3) below, and of linear combinations similar to (2). They have commonly employed the continuity and existence of derivatives of the coefficients. Under more lenient assumptions, the writer will obtain sufficient conditions that the zeros of the linear combination (2) shall be monotonic functions of $\mu$, the parameter. From this fundamental theorem, one may develop, with the less restrictive hypotheses, oscillation and existence theorems which extend many results obtained in the past for classical systems. $\ddagger$

We shall consider the linear differential system

$$
\begin{aligned}
& y_{x}^{\prime}(x, \mu)=K(x, \mu) z(x, \mu), \\
& z_{x}^{\prime}(x, \mu)=G(x, \mu) y(x, \mu),
\end{aligned}
$$

and the linear combination

$$
\phi(x, \mu)=\alpha(x, \mu) z(x, \mu)-\beta(x, \mu) y(x, \mu) .
$$

The following conditions shall be imposed:

Conditions S:

(a) $K$ and $G$ are summable $\S$ in $x$ on $X: a \leqq x \leqq b$, for every $\mu$ on $M$ : $M_{1}<\mu<M_{2} .|K(x, \mu)|,|G(x, \mu)| \leqq U(x) . \|$

(b) $\alpha, \beta$ are absolutely continuous in $x$ on $X$ for any fixed $\mu$ on $M$ and are continuous in $\mu$ for fixed $x$ on $X M,\left|\alpha_{x}^{\prime}\right|,\left|\beta_{x}^{\prime}\right| \leqq U(x)$ on $X M$.

(c) $K, G, \alpha_{x}^{\prime}$, and $\beta_{x}^{\prime}$ are continuous in $\mu$ for every $x$, on $X M$.

(d) $\alpha \neq 0$ on $\mathbb{X} X$.

* Presented to the Society, September 9, 1926; received by the editors in May, 1927.

$\dagger$ For references to the literature, see Ettlinger, these Transactions, vol. 19, p. 79.

$\ddagger$ The writer gratefully acknowledges Professor Ettlinger's helpful encouragement during the preparation of this paper.

\$ Throughout this paper, summable is used in the sense of Lebesgue.

\| The symbol $U(x)$ signifies some positive, summable function of $x$.

I We may actually admit, also, $\alpha \equiv 0$ on $X M$, for in this case condition (g) of $\mathrm{S}$ requires $\beta \neq 0$, and the zeros of $y$ and $\phi$ are coincident. We may then utilize a proof of Theorems B and II, stated in terms of $y$ instead of $\phi$. 
(e) $K(x, \mu)>0$ on* $X_{0} M$.

(f) $|y|+|z| \neq 0$ on $X M$.

(g) $\{\bar{\beta}\} \equiv\left(1 / \alpha^{2}\right)\{\alpha \beta\} \equiv\left(1 / \alpha^{2}\right)\left[\alpha \beta_{x}^{\prime}-\alpha_{x}^{\prime} \beta+\beta^{2} K-\alpha^{2} G\right]>c>0$ on $X_{0} M$ (or $<c<0$ on $\left.X_{0} M\right)$.

Concerning the system composed of (1) with the initial conditions

$$
y(a, \mu)=A(\mu), z(a, \mu)=B(\mu),
$$

where $A$ and $B$ are continuous in $\mu$ on $M$, we have the following

THEOREM A. There exists a unique pair of functions $(y, z)$, continuous in $(x, \mu)$ and absolutely continuous in $x$ on $X$ for $\mu$ fixed on $M$, which satisfy (1) on $X_{0} M$ and (3) on $M . \dagger$ (3).

Such a pair of functions, $(y, z)$, constitutes a solution of the system (1),

An implicit function theorem due to Ettlinger $\ddagger$ (and hereafter referred to as Theorem $\mathrm{B}^{\prime}$ ) will be employed in a modified form, as follows:

TheOREM B. Hypotheses:

(1) $f(\bar{x}, \bar{\mu})=0$, where $\bar{x}$ represents a set of values, $\bar{x}_{1}, \bar{x}_{2}, \cdots, \bar{x}_{n}$, on $X$ : $a \leqq x \leqq b$.

(2) $f(x, \mu)$ is absolutely continuous in $x$ on $X$ for any $\mu$ on $\bar{M}: \bar{M}_{1} \leqq \mu \leqq \bar{M}_{2}$. $f(x, \mu)$ is continuous in $\mu$ for fixed $x$ on $X M$.

(3) $f_{x}^{\prime}(x, \mu)$ is continuous in $\mu$ on $\bar{M}$ for any fixed $x$ on $X_{0} .\left|f_{x}^{\prime}\right| \leqq U(x)$ on $X \bar{M}$.

(4) $f_{x}^{\prime}(x, \mu)>0$ or $f_{x}^{\prime}(x, \mu)<0$ for almost every $x$ on an interval $|x-p| \leqq k$, $k>0$, when $\mu$ is fixed on $|\mu-m| \leqq h, h>0$, and whenever $(p, m)$ is a zero of $f$ on $X \bar{M}$. The numbers $k$ and $h$ may depend upon the particular $m$.

Conclusion: The function $x=x(\mu)$, such that $f[x(\mu), \mu] \equiv 0$, consists of a finite number of continuous and single-valued branches on $X \bar{M}$.

Consider first the case $n=1$. By Theorem $\mathrm{B}^{\prime}$, we have a continuous and single-valued function, $x=x(\mu)$, in and on the boundary of $D^{\prime} F^{\prime}$, which last is a neighborhood of the point $(\bar{x}, \bar{\mu})$ and is assumed wholly within $X \bar{M}$.

* $X_{0} M$ is to be read "at any fixed $\mu$ on $M$, for every $x$ on $X$ except possibly a set of measure zero."

$\dagger$ Tamarkin, Concerning some General Problems in the Theory of Ordinary Linear Differential Equations, etc., Petrograd, 1917, p. 29, ff., and Carathéodory, Vorlesungen über reelle Funktionen, Leipzig, 1918, p. 639, and p. 678. See also Ettlinger, Bulletin of the American Mathematical Society, vol. 33 , pp. $37-38$.

$\ddagger$ Existence theorems for implicit functions, Bulletin of the American Mathematical Society, vol. 34 , p. 316 . 
About the point $[x(\bar{\mu}+h), \bar{\mu}+h]$, on the boundary of $D^{\prime} F^{\prime}$, we may find a neighborhood into which and on the boundary of which $x(\mu)$ is continued, since all necessary hypotheses hold everywhere within $X \bar{M}$. The function is therefore continuous and single-valued to some $\mu$ outside of $D^{\prime} F^{\prime}$. We may continue to extend the definition of the function from $\bar{\mu}$ toward the right up to any other $\mu$ on $\bar{M}$, obtaining an $\operatorname{arc} A$. Due to a diminishing in the dimensions of the successively chosen neighborhoods, we may not be able to attain every $\mu$ on $\bar{M}$. Let $\mu=\mu^{*}$ be the lower bound of all values we cannot reach. We can approach arbitrarily close to $\mu^{*}$, and there is at least one point, $P:\left(x^{*}, \mu^{*}\right)$, which is a limit point of the set of zeros $A$. It follows, now, from a theorem which Ettlinger $\dagger$ has isolated from Caratheodory, that $f$ is a continuous function of $(x, \mu)$ when $x$ and $\mu$ vary independently on $X \bar{M}$; the zeros of $f$, accordingly, form a closed set in the same region. Then $f\left(x^{*}, \mu^{*}\right)=0$, and we may now obtain a neighborhood of $P$ in which $x=x(\mu)$ is single-valued and continuous. Since the set $A+P$ of zeros of $f$ is closed, the Heine-Borelł Theorem allows us to select a finite number of the covering neighborhoods to cover the set, and thence to obtain a finite number of overlapping elements of the continuous, single-valued curve, $x=x(\mu)$. Then this function is continuous and single-valued from $(\bar{x}, \bar{\mu})$ to $\left(x^{*}, \mu^{*}\right)$, where $\mu^{*}$ is any $\mu$ on $\bar{M}$.

Permit $n$ to be any integer, as assumed in Hypothesis (1). That this $n$, the number of zeros for $\mu$ fixed at some $\bar{\mu}$, always remains finite is an immediate consequence of the fourth hypothesis. For, if we assume an infinite set of zeros for any fixed $\bar{\mu}$, this set has a limit point $c$, and $f(c, \bar{\mu})=0$. But almost everywhere on some interval containing $c, f_{x}^{\prime}(x, \bar{\mu})>0$ or $<0$; therefore $(c, \bar{\mu})$ must be an isolated zero, and there is a contradiction. About each point $\left(\bar{x}_{i}, \bar{\mu}\right)$ we may draw a rectangular neighborhood $R_{i}$, and may trim each neighborhood in the $x$ direction, so that there is no overlapping in that direction. We may now construct for each of the finite number of zeros, just as was done for the single zero above, a function $x_{i}(\mu)$, continuous and single-valued on $\bar{M}$. None of the branches thus formed will intersect another on $\bar{M}$, for by Theorem $\mathrm{B}^{\prime}$ the distance between any two branches has a positive lower bound. In this case, therefore, the function $x(\mu)$ consists of a finite number of single-valued, continuous functions on $\bar{M}$.

With regard to a solution $y$ of the system (1), (3), S, we note immediately that the first three hypotheses of Theorem B are satisfied. Hypothesis (4) follows from conditions (e) and (f) of S, the continuity of $z$, and the first

† See footnote to Theorem A.

$\ddagger$ Cf. Hahn, Theorie der reellen Funktionen, Berlin, 1921, vol. 1, p. 89. 
equation of (1). Therefore, the function $x=x(\mu)$, representing the zeros of $y$, consists of a finite number of continuous, single-valued functions on $M$.

Consider $\psi=\phi / \alpha$, where $\phi$ is defined by (1), (2), and S. In the case of $\psi$, Hypotheses (2) and (3) of Theorem B are obviously valid for any $x$ on $X$, if $\mu$ is on any closed subinterval of $M$. We obtain the properties required in Hypothesis (4) through a

LEMma. If $K$ is bounded on $X$ for any fixed $\mu$ on $M$, and $\psi(\bar{x}, \bar{\mu})=0$, then $\psi_{x}^{\prime}(x, \bar{\mu})>g>0$ (or $<g<0$ ) almost everywhere on $|x-\bar{x}| \leqq k, k>0$, where $g$ is some constant.

Eliminating $z$ between

and

$$
\psi=z-\bar{\beta} y, \quad \bar{\beta}=\beta / \alpha,
$$

$$
\psi_{x}^{\prime}=-\bar{\beta} K z+y\left(G-\bar{\beta}_{x}^{\prime}\right)
$$

(which latter exists on $X_{0} M$ ), we obtain

$$
-\{\bar{\beta}\} y=K \bar{\beta} \psi-\psi_{x}^{\prime} \quad \text { on } X_{0} M,
$$

where

$$
\{\bar{\beta}\}=\bar{\beta}_{x}^{\prime}+K \bar{\beta}^{2}-G .
$$

Since $|y|+|z| \neq 0$ on $X M$, and since $y$ is continuous, we have either $y(x$, $\bar{\mu})<0$ or $y(x, \bar{\mu})>0$ on an interval $F:|x-\bar{x}| \leqq k^{*}$. For convenience, let it be negative on $F$. Actually, $y(x, \bar{\mu})$ has an extremum on $F$, and we may write $y(x, \bar{\mu})<-h<0, h$ being some constant. Given any positive $\eta$, there is, moreover, a sub-neighborhood, $F^{\prime}$, of $F$ such that $|\psi| \leqq \eta$ on $F^{\prime}$. Therefore, on setting $|K(x, \bar{\mu})|<K_{0}$ and $|\bar{\beta}(x, \bar{\mu})|<\bar{\beta}_{0}$ on $F^{\prime}$,

$$
\psi_{x}^{\prime}(x, \bar{\mu})>\{\bar{\beta}\} h-K_{0} \eta \bar{\beta}_{0},
$$

and hence, from condition $(\mathrm{g})$ of $\mathrm{S}$,

$$
\psi_{x}^{\prime}(x, \bar{\mu})>c h-K_{0} \eta \bar{\beta}_{0}=g>0 \text { on } F_{0}^{\prime},
$$

if $\eta$ is sufficiently small.

The cases where $y>0$ or $\{\bar{\beta}\}<c<0$ are treated in the same fashion.

But the zeros of $\phi$ and of $\psi$ are identical, and we may consequently assert that $x=x(\mu)$, the function such that $\phi[x(\mu), \mu] \equiv 0$, consists of a finite number of continuous, single-valued functions on any closed subinterval of $M$.

Comparatively simple conditions suffice to render the zeros of $\phi$ monotonic decreasing functions of $\mu$. They are embodied in Theorem II below, in preparation for which we shall prove Theorem I. $\dagger$

$\dagger$ Notation: All increments written $\delta f$ are changes in $f(x, \mu)$ due to change of $\mu$ alone. For brevity, only positive $\delta \mu$ 's are employed. 
THeOREM I. Hypotheses:

(1) $(y, z)$ is a solution of the system (1), (3), S.

(2) Either $A \equiv 0$ or $A \neq 0$ on $M$.

(3) For $\delta \mu>0,-\delta K, \delta G \leqq 0$, and if $A \neq 0, \delta(B / A) \leqq 0$. The three equalities do not hold simultaneously anywhere on $X M$. If $A \equiv 0,|\delta K|+|\delta G| \neq 0$ at any point of $X M$.

(4) $V(y, z)=y \delta z-z \delta y$.

Conclusion: $V(y, z)<0$ on $X M$.

The derivative of $V$ is

$$
V_{x}^{\prime}=y_{x}^{\prime} \delta z+y \delta z_{x}^{\prime}-z_{x}^{\prime} \delta y-z \delta y_{x}^{\prime} .
$$

By means of substitutions from (1), it may be written in a form given essentially by Sturm:*

$$
V{ }_{x}^{\prime}=\delta G\left(y^{2}+y \delta y\right)-\delta K \frac{y_{x}^{\prime 2}+y_{x}^{\prime} \delta y_{x}^{\prime}}{K(K+\delta K)} .
$$

Since $y$ and $y_{x}^{\prime}$ are continuous in $\mu$, given any $\eta>0$, there exists an $\epsilon_{\eta}>0$ such that whenever $\delta \mu \leqq \epsilon_{\eta}$, we have $|\delta y|,\left|\delta y_{x}^{\prime}\right| \leqq \eta$. Since, also, $|y|+\left|y_{x}^{\prime}\right|$ $>0$ almost everywhere, we may take $\delta \mu$ small enough that

$$
\operatorname{sgn} V_{x}^{\prime}=\operatorname{sgn}\left[y^{2} \delta G-y_{x}^{\prime 2} \frac{\delta K}{K(K+\delta K)}\right]=-1 \text { on } X M,
$$

save where $\delta G=\delta K=0$. We have $V(a, \mu)=A \delta B-B \delta A$, and

$$
\delta(B / A)=\frac{(A \delta B-B \delta A)}{A^{2}}\left(1-\frac{\delta A}{A+\delta A}\right) .
$$

For $\delta \mu$ small, again, and $A \neq 0$,

$$
\operatorname{sgn} \delta(B / A)=\operatorname{sgn} V(a),
$$

and $V(a, \mu) \leqq 0$ on $M$. If $A \equiv 0, V(a) \equiv 0$, and need not be considered. The conclusion of the theorem follows at once from the relation

$$
V(x, \mu)=\int_{a}^{x} V^{\prime}(t, \mu) d t+V(a, \mu) .
$$

TheOREM II. Hypotheses:

(1) $\phi(y, z)=\alpha(x, \mu) z(x, \mu)-\beta(x, \mu) y(x, \mu)$.

* Bocher, Leģons sur les Methodes de Sturm, Paris, 1917, p. 54. 
(2) $y$ and $z$ satisfy the system (1), (3), S on $X_{0} M$, with $\{\bar{\beta}\}>c>0$, and $\phi$ satisfies the conditions of $\mathrm{S} . K$ is bounded on $X$ for any fixed $\mu$ on $M$.

(3) $\delta(B / A) \leqq 0$ on $M$ if $A \neq 0 ;-\delta K, \delta G$, and $-\delta(\beta / \alpha) \leqq 0$ for any $x$ on $X$, when $\mu$ increases on $M$. Not all of the equalities hold simultaneously.

Conclusion: The zeros of $\phi$ move to the left with increase of $\mu$; i.e., if $\phi\left[x_{i}(\mu)\right.$, $\mu] \equiv 0, \delta x_{i}<0$ on $M$.

Let $\psi=\phi / \alpha=z-\bar{\beta} y, \bar{\beta}=\beta / \alpha$. Consider the function

$$
V\left(\psi, \psi_{x}^{\prime}\right)=\psi \delta \psi_{x}^{\prime}-\psi_{x}^{\prime} \delta \psi .
$$

Simplification by means of (1) gives us

$$
\begin{aligned}
V\left(\psi, \psi_{x}^{\prime}\right)=V & (y, z)\{\bar{\beta}\}+y^{2}\left(G \delta \bar{\beta}-\bar{\beta} \delta G+\bar{\beta} \delta \bar{\beta}_{x}^{\prime}-\bar{\beta}_{x}^{\prime} \delta \bar{\beta}\right) \\
& +z^{2}(-\bar{\beta} \delta K-K \delta \bar{\beta})+y z\left(\delta G-\delta \bar{\beta}_{x}^{\prime}+\bar{\beta}^{2} \delta K\right)+O\left(\delta \mu^{2}\right) .
\end{aligned}
$$

Here $O\left(\delta \mu^{2}\right)$ denotes additional terms of the second order in $\delta \mu$, such as $z \delta \bar{\beta} \delta y$, which, because of continuity, we can render as small as desired relative to first-order terms in $\delta \mu$ by choosing $|\delta \mu|$ sufficiently small.

Let $y$ and $z$ be solved for in terms of $\psi$ and $\psi_{x}^{\prime}$; the result is

$$
y=\frac{K \bar{\beta} \psi+\psi_{x}^{\prime}}{-\{\bar{\beta}\}}, \quad z=\frac{\psi\left(G-\bar{\beta}_{x}^{\prime}\right)+\bar{\beta} \psi_{x}^{\prime}}{-\{\bar{\beta}\}} .
$$

Since $\psi$ is continuous in $\mu$, if we let $\bar{x}=x_{i}(\bar{\mu})$ represent a zero of $\psi$, then given $\epsilon>0$, there exists $\eta_{\epsilon}>0$ such that whenever $|\mu-\bar{\mu}|<\eta_{\epsilon},\left|E_{1}\right|,\left|E_{2}\right|<\epsilon$, where we write

$$
y(\bar{x}, \mu)=\frac{\psi_{x}^{\prime}(\bar{x}, \mu)}{-\{\bar{\beta}\}}+E_{1}, z(\bar{x}, \mu)=\frac{\bar{\beta} \psi_{x}^{\prime}(\bar{x}, \mu)}{-\{\bar{\beta}\}}+E_{2} .
$$

Let these values be substituted into (4). On the same neighborhood, we find

$$
V\left[\psi(\bar{x}, \mu), \psi_{x}^{\prime}(\bar{x}, \mu)\right]=\left[V(y, z)\{\bar{\beta}\}-(1 /\{\bar{\beta}\}) \psi_{x}^{\prime 2}(x, \mu) \delta \bar{\beta}+O\left(\delta \mu^{2}\right)\right]_{x=\bar{x}} .
$$

But $\{\bar{\beta}\}>0$. Also, $-\delta \bar{\beta}=-\delta(\beta / \alpha) \leqq 0$, and $V(y, z)<0$ on $X M$. Therefore, on an interval $D:|\mu-\bar{\mu}|<\eta$, where $\eta \leqq \eta_{\epsilon}$,

$$
V\left[\psi(\bar{x}, \mu), \psi_{x}^{\prime}(\bar{x}, \mu)\right]<0 .
$$

If $x_{i}=x_{i}(\mu)$ represents the $i$ th zero of $\psi$ on $X$ for a certain $\mu$, and if $x_{1}=x_{i}\left(\mu_{1}\right)$, then the preceding inequality shows that in a neighborhood $D_{i}:\left|\mu-\mu_{1}\right|<\eta$,

$$
-\psi_{x}^{\prime}\left(x_{1}, \mu\right) \delta \psi\left(x_{1}, \mu\right)<0 .
$$

We set $x_{2}=x_{i}\left(\mu_{2}\right)=x_{1}+\delta x_{i}$, where $\delta \mu=\mu_{2}-\mu_{1}<\eta$, and may write 


$$
\int_{x_{1}}^{x_{1}+\delta x_{i}} \psi_{t}^{\prime}\left(t, \mu_{1}\right) d t=\psi\left(x_{2}, \mu_{1}\right) .
$$

Since $x_{i}(\mu)$ is continuous, by taking $\delta \mu$ small enough $\delta x_{i}$ may be made smaller than the distance between two consecutive zeros of $\psi\left(x, \mu_{1}\right)$ or $\psi\left(x, \mu_{2}\right)$. Then

$$
\begin{aligned}
\operatorname{sgn} \psi\left(x_{1}, \mu_{2}\right) & =-\operatorname{sgn} \psi\left(x_{2}, \mu_{1}\right) \\
& =\operatorname{sgn} \delta \psi\left(x_{1}, \mu\right) .
\end{aligned}
$$

The possibility that $\delta x_{i}=0$ is eliminated as an immediate consequence of a relation following from (5), namely,

$$
\delta \psi\left(x_{1}, \mu\right)=\psi\left(x_{1}, \mu_{2}\right) \neq 0 .
$$

From the Lemma, and from (5) and (7), it follows that in (6),

$$
\delta x_{i}<0 .
$$

The zeros of $\phi$ are identical with those of $\psi$, and hence they also move toward the left on $X$. with increase of $\mu$ on $M$.

By an argument very similar to the preceding one, employing $V(y, z)$ instead of $V\left(\psi, \psi_{x}^{\prime}\right)$, we may show independently of Theorem II that the zeros of $y$ (or of $z$ ) move to the left on $X$ as $\mu$ increases on $M$. When $\alpha \equiv 0$ on $X M$, the zeros of $\phi$ and of $y$ are identical; hence the conclusion of Theorem II is valid for $\alpha \equiv 0$ as well as for $\alpha \neq 0$ on $X M$.

The writer has employed Theorem II to obtain existence and oscillation theorems for the Sturmian and for the general self-adjoint systems under the hypotheses of $S$ concerning continuity and existence of derivatives. The methods follow, in general, those of Bôcher* and of Ettlinger, $\dagger$ and the results of Ettlinger concerning the distribution of characteristic numbers, etc., may be duplicated.

- Bócher, loc. cit.

† Ettlinger, these Transactions, vol. 19, pp. 79-96, and vol. 22, pp. 136-143.

UNIVERSITY OF TEXAS,

Austin, TeXas 